\title{
Meshless methods with application to metal forming
}

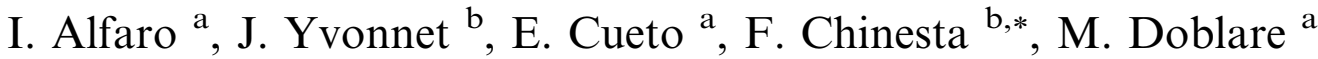 \\ a Aragón Institute for Engineering Research (I3A), University of Zaragoza, María de Luna, 3, E-50018 Zaragoza, Spain \\ b LMSP, UMR 8106 CNRS ENSAM-ESEM, 151 boulevard de l'Hopital, F-75013 Paris, France
}

\begin{abstract}
In recent years, a new family of computational methods has emerged. The so-called meshless or mesh-free methods have been inves-tigated and used by many researchers for treating a large variety of engineering problems, involving usually large displacements as encountered for example in forming process simulations. The main advantage of meshless methods is the fact that the interpolation accu-racy is not significantly affected by the nodal distribution. Nevertheless, a troublesome task in these techniques is the imposition of essen-tial boundary conditions, as in general the Kronecker delta property is not satisfied. Despite of several techniques have been proposed to overcome this problem, an appealing alternative technique which allows to circumvent this difficulty lies in the use of the natural neigh-bor interpolation (NEM). This paper focuses on the description and analysis of the natural element method, and its application for sim-ulating some forming processes involving large displacements as well as fixed or moving discontinuities.
\end{abstract}

Keywords: Meshless methods; Natural neighbor Galerkin method; $\alpha$-shapes; Constrained natural element method

\section{Introduction}

In recent years, a new family of computational methods has emerged. The so-called meshless or mesh-free methods have been investigated and used by many researchers for treating a large variety of engineering problems, involving usually large displacements as encountered for example in forming process simulations (free surface or moving boundary problems, moving interfaces, cracks propagation, etc.). In these problems accurate finite element solutions require significant computational efforts in remeshing steps. In contrast, meshless methods require only nodal data without explicit connectivity between nodes.

The finite element method (FEM), which has been widely used in many engineering problem simulations, exhibits some limitations as the interpolation fails when the elements become too distorted [1]. The main advantage of meshless methods is the fact that the interpolation accuracy is not significantly affected by the nodal distribution. However, it is obvious that in any case, an appropriate nodal density is required in order to describe high gradients (boundary layers) as well as an anisotropic behavior of the solution. For this reason, nodal adaptation is needed to compute numerical solutions of problems governed by partial differential equations.

In the framework of the finite elements, these adaptation procedures are known as remeshing techniques. Remeshing is required for example when the element geometry becomes too distorted as a consequence of large domain changes. Moreover, sometimes, in order to improve the interpolation accuracy for describing boundary layers or an anisotropic behavior,

\footnotetext{
Research partially supported by the Spanish Ministry of Science and Technology: project CICYT-DPI2002-01986.

* Corresponding author. Tel.: +33 144246371 ; fax: +33 144246468 .

E-mail address: francisco.chinesta@paris.ensam.fr (F. Chinesta).
} 
new nodes must be added, removed or repositioned, and in fact this is not an easy task because the mesh associated to the new nodal distribution cannot contain any too distorted element. If this is not the case, local or global remeshing is required in order to guarantee the geometrical quality of the mesh elements.

On the contrary, in meshless techniques, interpolation is free of that mesh requirement. Thus, introduction, eliminations or repositioning of nodes is a trivial task, because no geometrical restrictions exist. In this way, nodes can be added without geometrical checks in the regions where the solution must be improved (identified by using an appropriate error indicator). Once that the new nodes are placed into the domain, and for problems making use of internal variables, these variables can be initialized at those nodes using the standard meshless interpolation. This appealing feature of this kind of techniques simplifies significantly the refinement procedures.

Many meshless methods have been developed, including the smooth particle hydrodynamics (SPH) [2], the diffuse element method (DEM) [3], the partition of unity method (PUM) [4], the h-p clouds method [5], the element free Galerkin (EFG) method [6], the reproducing kernel particle method (RKPM) [7], among many others. Nevertheless, a troublesome task in these techniques is the imposition of essential boundary conditions, because in general the Kronecker delta property is not satisfied. Despite of several techniques have been proposed to overcome this problem [6,8], an appealing alternative technique which allows to circumvent this difficulty lies in the use of the natural neighbor interpolation $[9,10]$.

The natural neighbor Galerkin method, or natural element method (NEM), which has been considered as a member of the meshless methods family, satisfies the Kronecker delta property as well as the linear consistency (which implies the partition of unity). Sukumar has shown in [10] the strict linearity of the shape functions along any convex boundary. However, on the non-convex ones, the NEM shape functions are no more linear. In this way, if we consider a point located on a nonconvex boundary, the interpolated value of a field at this position depends on the two neighbor nodes located on the boundary and on other spurious nodes, some located in the neighborhood of this point within the domain and others on the non-convex boundary far from the considered point, as described in our former works $[11,12]$.

A strategy to overcome this problem and to recover the linearity of the shape functions along any kind of boundary (convex or not) was proposed by Cueto et al. in [11] by invoking the concept of $\alpha$-shapes. Moreover, this technique allows to proceed without an explicit description of the domain boundary, which is automatically extracted from the cloud of nodes. To describe accurately some geometrical details, an appropriate adaptation of the nodal density is required.

If the domain boundary is defined using a CAD description, the shape functions can be computed from the constrained Voronoi diagram instead of the Voronoi diagram used in the NEM, without any nodal density control requirement. It has been proved in some of our former works that the use of this technique, known as the constrained natural element method (C-NEM), allows to recover the shape functions linearity along any kind of boundary. Despite of the constrained Voronoi diagram makes use of a visibility criterion, no numerical discontinuities are introduced. Thus, cracks and fixed or moving discontinuities can be treated in a very simple manner, as proved in $[12,13]$.

The partition of unity (PU) framework $[4,14]$ is a powerful technique to enrich standard functional approximations. The main idea is to extend the usual approximation considering the product of the standard shape functions by local or global enrichment functions. The extended finite element method (X-FEM) is a variation on this framework. Recently, the $\mathrm{X}$-FEM has been coupled to the level set method [15] to represent interface topologies [16]. In this way, the discontinuity evolution can be properly represented on a fixed background mesh, just by adding an appropriate enrichment in the functional approximation in the elements concerned by the moving discontinuity [17]. However, when the material in which the interface is moving is subjected to large displacements, an updated Lagrangian description could be suitable. Thus, when the background mesh evolves, remeshing will be required also to avoid too high background mesh distortions.

Level set and fast marching methods (FMM) [18] are well-known interface-capturing techniques in which the interface is represented as the zero level contour of a function (level set) of one higher-dimension. The coupling of partition of unity techniques to level set methods is an appealing means to carry out geometric computations, evaluate enrichment functions (especially in 3D), and to evolve interfaces on a fixed or also moving background cloud of nodes. Those capturing techniques seems to be more suitable that the tracking ones to describe large interface evolutions.

Moreover, the PU paradigm results very useful to discretize mixed variational formulations in the natural elements framework. In this way, Gonzalez et al. [19] have used a global enrichment of the displacement field approximation to satisfy the LBB condition in the incompressible elasticity limit.

The next sections deal with the description and analysis of the natural element method (NEM). In Section 2 we discuss methods based on natural neighbor interpolation, the so-called natural neighbor Galerkin or natural element methods. Different approaches to enforce essential boundary conditions in these methods exist. In Section 2.2 we describe the approach proposed by Cueto et al. [11] based on the use of $\alpha$-shapes. In Section 2.3 we describe the technique which derives from the introduction of a visibility criterion [12]. Finally, in Section 3, we analyze some applications of these strategies in forming process simulations. The $\alpha$-NEM will be applied in the simulation of aluminium extrusion, where its convenience will be deeply discussed. On the other hand, a metal casting problem involving a moving discontinuity (material interphase) will be treated by using the C-NEM (constrained natural element method) which seems to be specially suitable in this kind of problems. 


\section{Methods based on natural neighbor interpolation}

In this section, after a brief review of the Voronoi-based interpolants, we introduce the technique based on the use of the $\alpha$-shapes, as well as another based on the use of the constrained Voronoi diagram.

\subsection{Natural neighbor interpolation}

We briefly touch upon the foundation of Sibson's natural neighbor coordinates (shape functions) that are used in the natural element method. For a more in-depth discussion on the Sibson interpolant and its application for solving secondorder partial differential equations, the interested reader can refer to Sambridge et al. [9], and Sukmar et al. [10]. The NEM interpolant is constructed on the underlying Voronoi diagram. The Delaunay tessellation is the topological dual of the Voronoi diagram.

Consider a set of nodes $S=\left\{n_{1}, n_{2}, \ldots, n_{N}\right\}$ with coordinates $\left\{\mathbf{x}_{1}, \mathbf{x}_{2}, \ldots, \mathbf{x}_{N}\right\}$, assumed by simplicity in $\mathfrak{R}^{2}$. The Voronoi diagram is the subdivision of $\mathfrak{R}^{2}$ into regions $T_{i}$ (Voronoi cells) defined by

$$
T_{i}=\left\{\mathbf{x} \in \mathfrak{R}^{2}: d\left(\mathbf{x}, \mathbf{x}_{i}\right)<d\left(\mathbf{x}, \mathbf{x}_{j}\right), \forall j \neq i\right\} \quad \forall i .
$$

The Sibson coordinates of $\mathbf{x}$ with respect to a natural neighbor $n_{i}$ (see Fig. 1) is defined as the ratio of the overlap area (volume in 3D) of their Voronoi cells to the total area (volume in 3D) of the Voronoi cell related to point $\mathbf{x}$ :

$$
\phi_{i}(\mathbf{x})=\frac{\text { Area }(\text { afghe })}{\text { Area }(\text { abcde })} .
$$

If the point $\mathbf{x}$ coincides with the node $n_{i}$, i.e. $\mathbf{x}=\mathbf{x}_{i}, \phi_{i}\left(\mathbf{x}_{i}\right)=1$, and all other shape functions vanish, i.e. $\phi_{j}\left(\mathbf{x}_{i}\right)=\delta_{i j}\left(\delta_{i j}\right.$ being the Kroenecker delta). The properties of positivity, interpolation, and partition of unity are then verified [10]:

$$
\left\{\begin{array}{l}
0 \leqslant \phi_{i}(\mathbf{x}) \leqslant 1, \\
\phi_{i}\left(\mathbf{x}_{j}\right)=\delta_{i j}, \\
\sum_{i=1}^{n} \phi_{i}(\mathbf{x})=1 .
\end{array}\right.
$$

The natural neighbor shape functions also satisfy the local coordinate property [20], namely

$$
\mathbf{x}=\sum_{i=1}^{n} \phi_{i}(\mathbf{x}) \mathbf{x}_{i}
$$

which combined with the partition of unity (Eq. (3)), implies that the natural neighbor interpolant spans the space of linear polynomials (linear completeness).

Natural neighbor shape functions are $C^{\infty}$ at any point except at the nodes, where they are only $C^{0}$, and on the boundary of the Delaunay circles (spheres in $3 \mathrm{D}$ ) where they are only $C^{1}$, because of the discontinuity in the neighbors nodes across these boundaries. Hiyoshi and Sugura [21] have shown that the Sibson interpolant belongs to a more general class of Voronoi-based interpolants, called $k$ th order standard coordinates, proving that the interpolant generated by the $k$ th order standard coordinates have $C^{k}$ continuity on the Delaunay circles (spheres) boundaries. In this context, the Sibsonian and non-Sibsonian (Laplace) coordinates [22] result to be the standard coordinates of order 1 and 0 , respectively.

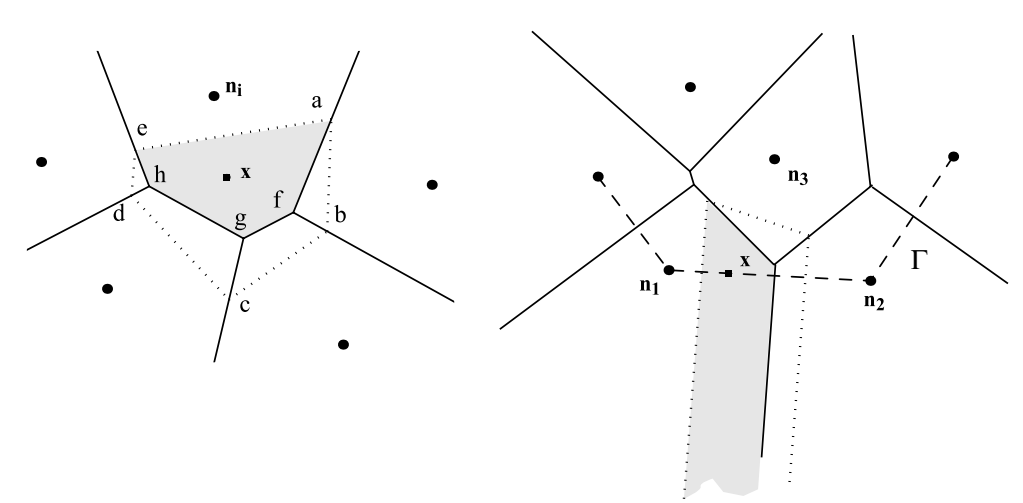

Fig. 1. Construction of the Sibson shape functions: (left) evaluating the shape function related to node 1 at point $x$ (typically an integration point); (right) evaluating the shape function at a point located on the domain boundary. 
Another important property of this interpolant is the ability to reproduce linear functions over the boundary of convex domains. The proof can be found in Sukumar et al. [10]. An illustration is depicted in the right part of Fig. 1: as the areas associated to points on the boundary become infinite, the contribution of internal points vanish in the limit when the point approaches the convex boundary, and the shape functions associated with nodes $n_{1}$ and $n_{2}$ become linear on the segment $\left(n_{1}-n_{2}\right)$. This is not true in the case of non-convex boundaries, and the next subsections focus in two approaches to circumvent this difficulty.

If one considers an interpolation scheme for a scalar (vectorial or tensorial) function $T(\mathbf{x}): \Omega \subset \mathfrak{R}^{2} \rightarrow \mathfrak{R}^{2}$, in the form

$$
T^{h}(\mathbf{x})=\sum_{i=1}^{n} \phi_{i}(\mathbf{x}) T_{i}
$$

where $T_{i}$ are the nodal variables related to the $n$ natural neighbor nodes of point $\mathbf{x}$, and $\phi_{i}(\mathbf{x})$ are the shape functions associated with each neighbor node, then it can be noted that Eq. (5) defines a local interpolation scheme. Thus, the trial and test functions used in the discretization of the variational formulation describing the models considered in this paper take the form of Eq. (5).

\subsection{The $\alpha$-shapes based natural element method ( $\alpha$-NEM)}

One important issue when using meshless methods, and particularly when one simulates forming processes from an updated Lagrangian formulation, is free surface tracking. Since, by definition, meshless methods do not need any explicit connectivity between nodes, and consequently the nodes belonging to the domain boundary must be identify with the help of an appropriate technique. We can proceed, as in the finite elements framework, by marking the nodes that belong to the boundary. However, in any case, special treatments are needed when for example a bubble is generated, when two or several moving fronts interact, or when the nodes approach to solid walls. In this section a different approach will be introduced. This approach is based on the use of the geometrical concept of $\alpha$-shapes. Edelsbrunner and co-workers established the concept of the family of $\alpha$-shapes of a cloud of points when dealing with the problem of finding the shape of a cloud of points. The concept of shape had traditionally no formal meaning, so they rigourously defined a complete family of shapes of a cloud of points by introducing a parameter $\alpha$ that can be considered as a measure of the level of detail up to which the domain is going to be represented.

A cloud of points itself (without any connectivity between them, nor explicit definition of the boundary) defines a finite number of shapes. For a proper definition of the concept of shape, Edelsbrunner [23] established the definition of a complete family of shapes of a cloud of points, based on the Delaunay triangulation (tetrahedralization in $\mathfrak{R}^{3}$ ), that is unique for a given cloud.

In essence, an $\alpha$-shape is a polytope that is not necessarily convex nor connected. It is triangulated by a subset of the Delaunay triangulation of the nodes, and hence the empty circumcircle criterion holds. Let $N$ be a finite set of points in $\mathfrak{R}^{3}$ and $\alpha$ a real number, with $0 \leqslant \alpha<\infty$. A $k$-simplex $\sigma_{T}$ with $0 \leqslant k \leqslant 3$ is defined as the convex hull of a subset $T \subseteq N$ of size $|T|=k+1$. Let $b$ be an $\alpha$-ball, i.e., an open ball of radius $\alpha$. A $k$-simplex $\sigma_{T}$ is said to be $\alpha$-exposed if there exist an empty $\alpha$-ball $b$ with $T=\partial b \cap N$ where $\partial \Omega$ refers to the boundary of the ball. In other words, a $k$-simplex is said to be $\alpha$-exposed if an $\alpha$-ball that passes through its defining points contains no other point of the set $N$.

We can now define the family of sets $F_{k, \alpha}$ as the sets of $\alpha$-exposed $k$-simplexes for the given set $N$. This allows us to define an $\alpha$-shape of the set $N, \mathscr{S}_{\alpha}(N)$, as the polytope whose boundary consists of the triangles in $F_{2, \alpha}$, the edges in $F_{1, \alpha}$ and the vertices or nodes in $F_{0, \alpha}$. As remarked before, an $\alpha$-shape is a polytope that can be triangulated by a subset of the Delaunay triangulation or tetrahedralization.

In order to complete the definition of an $\alpha$-shape, it remains to be defined which part of $\mathfrak{R}^{n}-\partial \mathscr{S}_{\alpha}$ is interior or exterior to the boundary. Notice that for each $\alpha$-exposed triangle $\sigma_{T}$ (in 3D) there are two (not necessarily empty) $\alpha$-balls $b_{1}$ and $b_{2}$ so that $T \subseteq \partial b_{1}$ and $T \subseteq \partial b_{2}$. If both balls are empty, then $\sigma_{T}$ does not belong to the boundary of the interior of $\mathscr{S}_{\alpha}$. Assume now that $b_{1}$ is empty and $b_{2}$ not. In this case, $\sigma_{T}$ bounds the interior of $\mathscr{S}_{\alpha}$ (the interior of $\mathscr{S}_{\alpha}$ and the center of $b_{1}$ lie on different sides of $\sigma_{T}$ ).

It can be easily demonstrated that the following relationships holds for the Delaunay triangulation of the cloud and its $\alpha$-shapes:

$$
F_{k}=\bigcup_{0 \leqslant \alpha<\infty} F_{k, \alpha} \text { for } 0 \leqslant k \leqslant 2,
$$

where $F_{k}$ represents the set of $k$-simplexes bounded by an empty ball (that is, the Delaunay triangulation of the set).

This definition of the shape of a cloud of points allows us to dynamically extract the shape of the cloud of points as it evolves during a material deformation process. If there exists a proper relationship between the $\alpha$ value and the nodal distance, $h$-in the sense that $\alpha$ is a measure of the level of detail up to which the domain is represented, and must be of the order of $h$ - the mass conservation can be guaranteed [24]. 


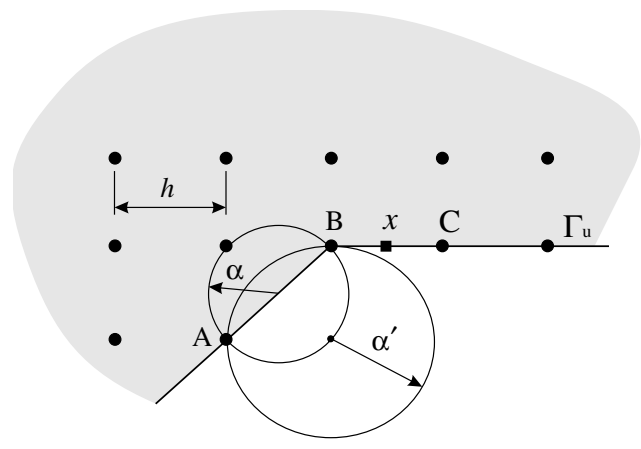

Fig. 2. Neighbourhood in the context of $\alpha$-complexes.

In order to clarify the influence of $\alpha$-shapes in the computation of the shape functions, consider a two-dimensional (extension to 3D is straightforward) regular gridded set of $N$ nodes along a non-convex boundary $\Gamma_{u}$ as depicted in Fig. 2. Let $h$ be the nodal spacing, and the minimum value $\alpha$ to achieve a proper reproduction of the geometry is $\alpha=\frac{\sqrt{2}}{2} h$. Consider a point $\boldsymbol{x}$ that belongs to $\Gamma_{u}$ : for a proper imposition of essential boundary conditions, this point must have only two neighbors on the boundary, namely nodes $B$ and $C$. For this to occur, the node $A$ must not be a natural neighbor of $\boldsymbol{x}$ (and thus triangle $A B C$ must not belong to the $\alpha$-shape used to represent the domain). The worst case occurs when $\boldsymbol{x}$ tends to $B$. The $\alpha$-ball that would make the points $A, B$ and $\boldsymbol{x}$ pertain to $\mathscr{C}_{\alpha}(N \cup \boldsymbol{x})$ would have a radius $\alpha^{\prime}=h>\alpha$. This is valid whenever the angle formed by the segments $\overline{A B}$ and $\overline{B C}$ is less than $90^{\circ}$. For lower angles it is necessary to explicitly define the boundary, trough some CAD technique.

In order to clarify the concepts just introduced, we present in the following paragraphs some examples of $\alpha$-shapes computed from a cloud of points corresponding to the simulation of an extrusion process. In this section we will restrict ourselves to geometrical concepts only.

Consider the extrusion example shown in Fig. 3, where the equivalent plastic strain rate field is depicted. The key idea of the method here proposed is to extract the shape of the domain at each time step by invoking the concept of $\alpha$-shape of the cloud. The $\alpha$ parameter will be obtained by geometrical considerations. In this case the radius at the outlet of the tooling, for instance, seems to be the smallest level of detail up to which the domain must be represented. The nodal distance $h$ must be accordingly chosen.

In Fig. 4 some members of the family of $\alpha$-shapes of the cloud of points in its final configuration (corresponding to Fig. 3(b)) are depicted. In Fig. 4(a) the member for $\alpha=0$, i.e., the cloud of points itself, is shown. Note how, as $\alpha$ is increased, the number and size of the simplexes (in this case, triangles) that belong to the shape is increasing. For $\alpha=1.0$ we obtain an appropriate shape for the cloud. Note, however, that this is not an exact value to be determined at each time step. There exists an interval of acceptable $\alpha$ values for a single shape. Finally, by increasing the $\alpha$ value, the convex hull of the cloud of points is reached (Fig. 4(f)).

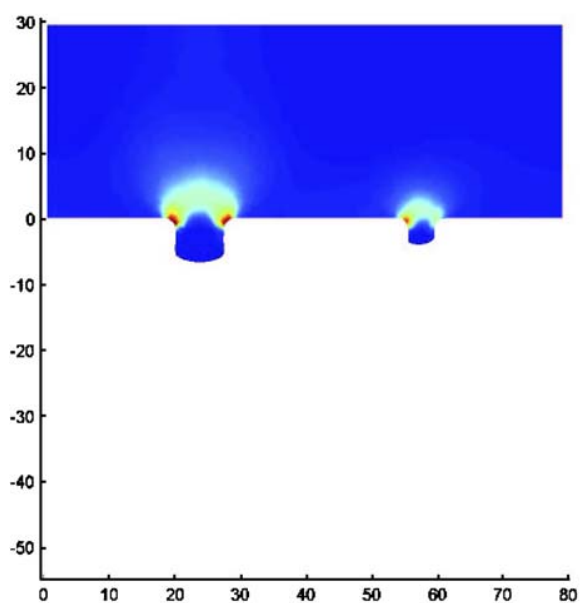

(a)
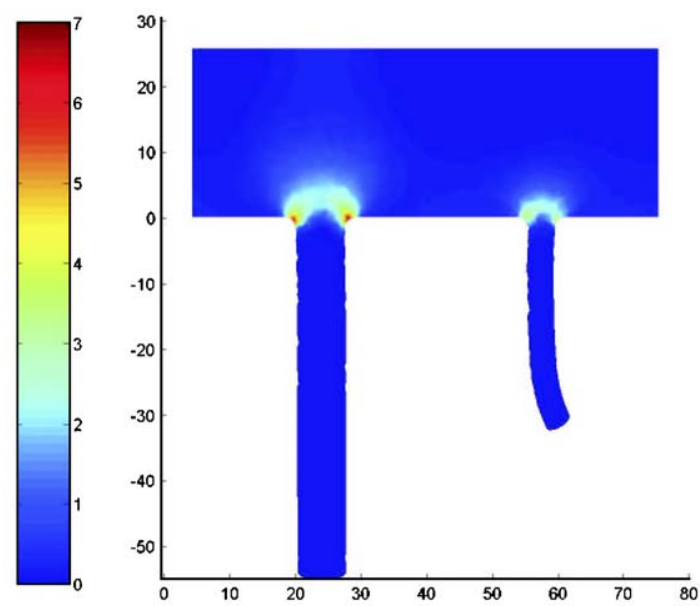

(b)

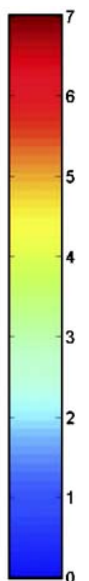

Fig. 3. Equivalent plastic strain rate at two instants of the extrusion process. 


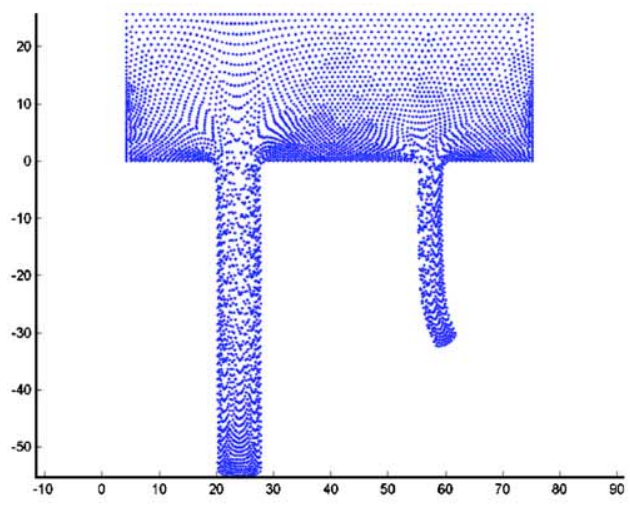

(a)

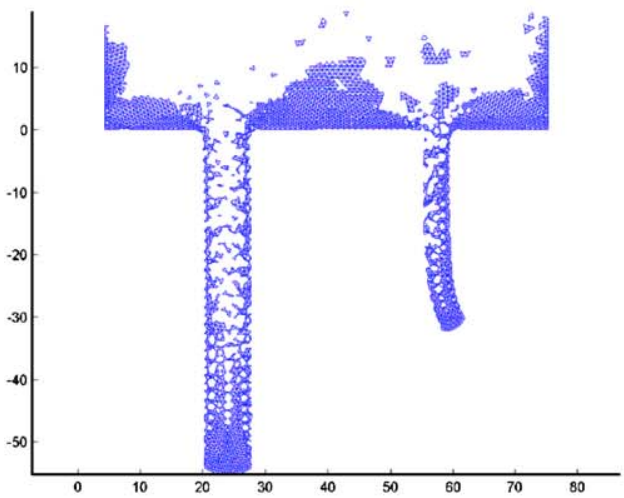

(c)

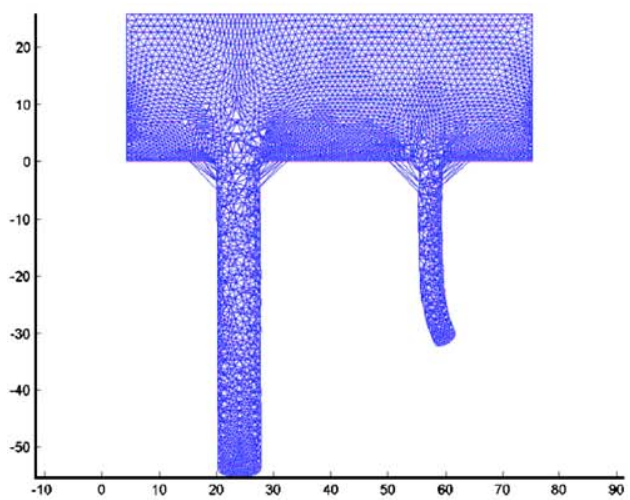

(e)

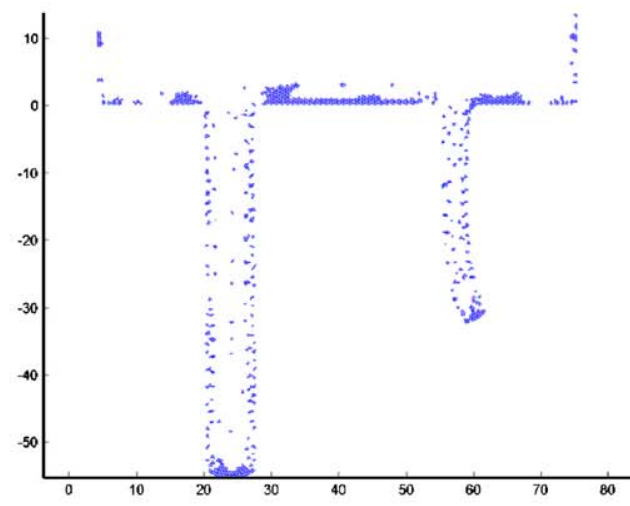

(b)

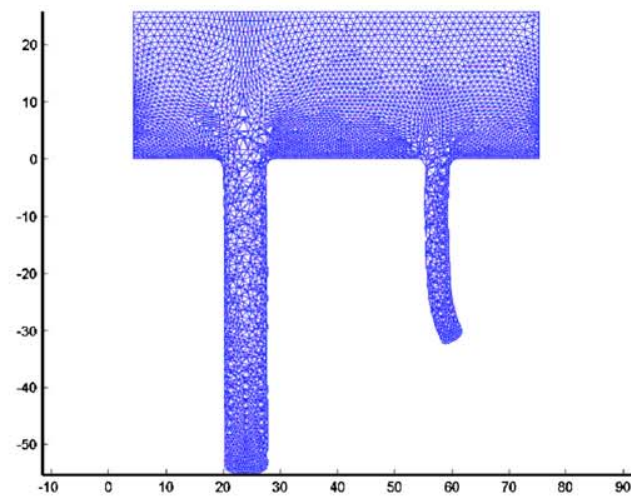

(d)

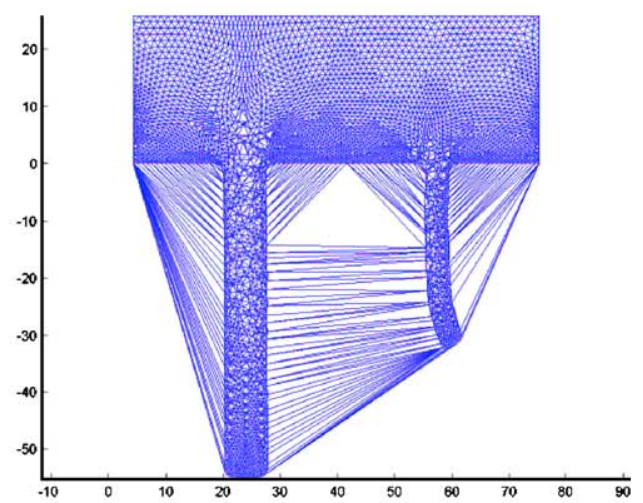

(f)

Fig. 4. Some members of the family of $\alpha$-shapes of the cloud of points used in the extrusion example. (a) $\mathscr{S}_{0}$ (the cloud of points), (b) $\mathscr{S}_{0.3}$, (c) $\mathscr{S}_{0.5}$, (d) $\mathscr{S}_{1.0}$, (e) $\mathscr{S}_{1.5}$ and (f) $\mathscr{S}_{\infty}$ (the convex hull of the set).

The extraction of the shape of the domain throughout the time interval of the simulation is not the only application of $\alpha$-shapes in the approach here presented. In fact, in Cueto et al. [11], it was demonstrated that the ability of natural neighbor interpolation to reproduce linear polynomials over convex regions of the boundary can be extended to the non-convex ones. This can be achieved by restricting natural neighbor interpolation to nodes that are neighbors, in the $\alpha$-shape sense, that is, are connected by an $\alpha$-exposed simplex.

This makes the natural element method one of the few meshless methods that can reproduce exactly linear polynomials over the boundary of any domain. When dealing with piece-wise homogeneous domains, for instance, it is also necessary to ensure the discontinuity of the derivatives of some field (which is itself continuous across the interface). This can also be done by avoiding natural neighborhood between nodes placed at both sides of the material interface, as demonstrated in [25].

This particular implementation of the NEM received the name of $\alpha$-shape based NEM (or, simply $\alpha$-NEM). Its performance in the simulation of aluminium extrusion will be analyzed in Section 3.1. 


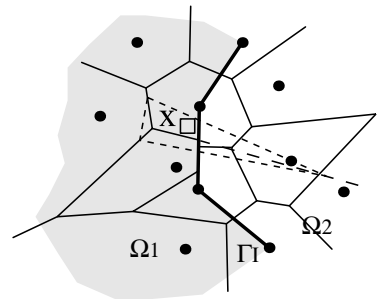

(a)

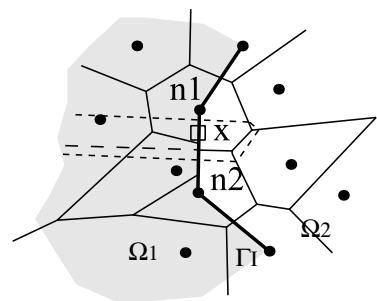

(b)

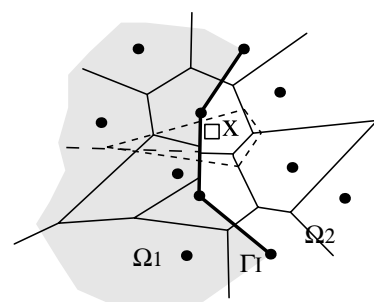

(c)

Fig. 5. Reproducing discontinuous derivatives using the constrained Voronoi diagram.

\subsection{The constrained natural element method (C-NEM)}

It was proved in [10-12] that spurious influences between non-visible nodes and lost of linearity in the interpolation along boundaries of non-convex domains appear in the framework of the NEM. In order to avoid this drawback and to recover all the appealing properties of the method for any geometry (including non-convex domains containing cracks or involving field discontinuities), a visibility criterion is introduced in order to restrict influent nodes among natural neighbors.

Thus, the resulting constrained Vornoi diagram (CVD) is composed with cells $T_{i}^{\mathrm{C}}$, one for each node $n_{i}$, such that any point $x$ inside $T_{i}^{\mathrm{C}}$ is closer to $n_{i}$ than to any other node $n_{j}$ visible from $x$. The constrained Voronoi cells are defined formally by

$$
T_{i}^{\mathrm{C}}=\left\{\mathbf{x} \in \mathfrak{R}^{n}: d\left(\mathbf{x}, \mathbf{x}_{i}\right)<d\left(\mathbf{x}, \mathbf{x}_{j}\right), \forall j \neq i, S_{x \rightarrow n_{i}} \cap \Gamma=\emptyset, S_{x \rightarrow n_{j}} \cap \Gamma=\emptyset\right\},
$$

where $\Gamma$ is the domain boundary and $S_{a \rightarrow b}$ denotes the segment between the points $a$ and $b$.

A generalization of the constrained Delaunay triangulation to 3D does not exist without adding new nodes. Nevertheless, some techniques for constructing 3D constrained Delaunay tessellations are available and provided in [26,27] by addition of Steiner points.

In order to solve partial differential equations defined in non-convex domains, or to reproduce some functional discontinuities, we consider the following approximation of both the trial and the test functions:

$$
T^{h}(\mathbf{x})=\sum_{i=1}^{V} \phi_{i}^{\mathrm{C}}(\mathbf{x}) T_{i}
$$

where $V$ is the number of natural neighbors visible from point $\mathbf{x}$ and $\phi_{i}^{\mathrm{C}}$ is the constrained natural neighbor shape function related to the $i$ th node at point $x$. The computation of the $\mathrm{C}-\mathrm{n}-\mathrm{n}$ (constrained natural neighbor) shape functions is similar to the natural neighbor shape function, when one proceed using the constrained Voronoi diagram introduced previously. It was shown in $[13,12]$ that the use of the constrained Voronoi diagram does not affect the properties of the NEM interpolation, allowing the extension of the linearity of the shape functions on the convex domain boundaries, to any geometry, convex or not.

The ability of the C-NEM for treating problems involving cracks has been illustrated in [12]. In the present paper, we focus on its application in the context of a moving interface defining two domains with different thermal properties. Thus, defining at time $t$ two CVD (constrained Voronoi diagrams) of $\Omega_{1}(t)$ and $\Omega_{2}(t)$, both with respect to the interface $\Gamma_{\mathrm{I}}(t)$, it can be proved that the interpolated temperature field is $C^{\infty}$ everywhere, except at the nodes and on the interface $\Gamma_{\mathrm{I}}(t)$ where it is only $C^{0}$ and on the Delaunay circles where it is $C^{1}$. Thus, this interpolation seems to be appropriate to simulate the Stefan problem considered in this paper.

To illustrate this behavior, we consider the situation depicted in Fig. 5, where the point $\mathbf{x}$ moves from $\Omega_{1}$ to $\Omega_{2}$. If $\mathbf{x}$ is in $\Omega_{1}$, the interpolated field is constructed from Eq. (8) using the neighbor visible nodes from point $\mathbf{x}$ ( $\Gamma_{\mathrm{I}}$ is assumed opaque). If $\mathbf{x}$ is on $\Gamma_{\mathrm{I}}$, according to the previous discussion, the interpolated field is strictly linear because it only depends on the two neighbor nodes located on $\Gamma_{\mathrm{I}}$. Finally, when $\mathbf{x}$ is in $\Omega_{2}$, the interpolated field is defined using the visible neighbor and visible nodes from point $\mathbf{x}$ ( $\Gamma_{\mathrm{I}}$ being opaque). The continuity of the interpolated field is then guaranteed, but a discontinuity appears in the field derivatives, because of a sudden change in the neighbor nodes across the interface. We can then reproduce the temperature field continuity, as well as the expected flux discontinuity on the interface.

\section{Numerical examples}

In this section, we illustrate some applications of both natural elements strategies in forming process simulations. The $\alpha$-NEM will be applied in the simulation of aluminium extrusion, where its convenience will be deeply discussed. A casting 
problem involving a moving interphase will be simulated by using the C-NEM which seems to be specially adapted for treating this kind of problems.

\subsection{Aluminium extrusion simulation using the $\alpha-N E M$}

As it is well known, extrusion is a forming process consisting in the production of straight, long profiles by pressing a billet of hot aluminium or other metal through a hole with a certain shape. From a numerical point of view, it involves the interaction of complex problems, such as thermo-plasticity, large deformations, contact and free surface development. In this example we will analyze the $\alpha$-NEM ability for simulating such processes.

We consider a simple two-dimensional geometry for the extrusion die, as shown in Fig. 6. Due to the symmetry of the die, only one half of the extrudate was considered and appropriate boundary conditions were applied.

The flow of the billet is assumed to be governed by the following equations:

- The balance of momentum equations, without inertia and mass terms

$$
\nabla \cdot \boldsymbol{\sigma}=\mathbf{0},
$$

where $\sigma$ is the stress tensor.

- The incompressibility condition

$$
\nabla \cdot \mathbf{v}=0,
$$

where $\mathbf{v}$ represents the velocity field.

- The aluminium behavior is governed by a (rigid) visco-plastic law:

$$
\boldsymbol{\sigma}=-p \mathbf{I}+\left(\frac{2 \bar{\sigma}}{3 \dot{\kappa}}\right) \mathbf{D}
$$

where $\mathbf{D}$ represents the strain rate tensor. The flow stress is given by a modified Sellars-Tegart law [28,29]

$$
\bar{\sigma}(\dot{\kappa}, T)=s_{\mathrm{m}} \operatorname{arcsinh}\left[\left(\frac{\dot{\kappa}+\dot{\kappa}_{0}(T)}{A} \exp \left(\frac{Q}{R T}\right)\right)^{1 / n}\right]
$$

which takes into account the effect of temperature on the flow stress. $\dot{\kappa}$ represents the equivalent plastic strain rate, i.e., $\dot{\kappa}=\sqrt{\frac{2}{3} \mathbf{D}: \mathbf{D}} \cdot \dot{\kappa}_{0}$ is a temperature-dependent parameter added to the Sellars-Tegart law in order to ensure the flow stress to be greater than zero at zero plastic deformation [29]. $s_{\mathrm{m}}$ and $n$ are strain rate sensitivity parameters, $Q$ is
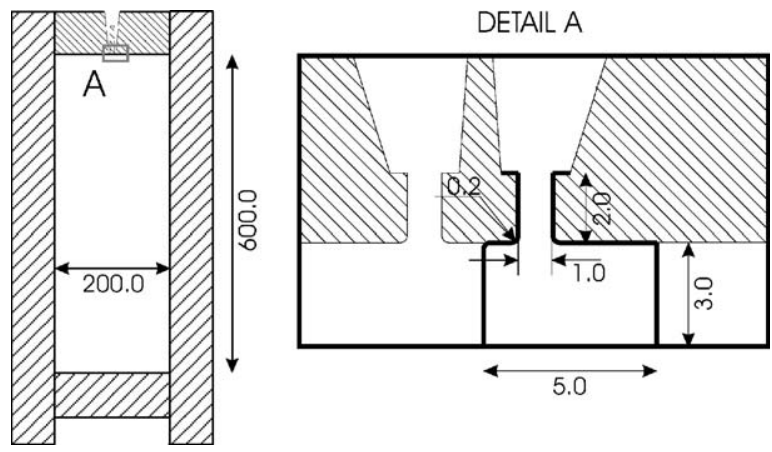

Fig. 6. Geometry of the extrusion die and detail of the simulated region.

Table 1

Material parameters for AA6063 aluminium alloy [29]

\begin{tabular}{ll}
\hline Parameter & Value \\
\hline$\dot{\kappa}_{0}\left(\mathrm{~s}^{-1}\right)$ & 0.005 \\
$s_{\mathrm{m}}(\mathrm{MPa})$ & 25 \\
$n$ & 5.4 \\
$A\left(\mathrm{~s}^{-1}\right)$ & $6 \times 10^{9}$ \\
$Q(\mathrm{~J} / \mathrm{mol})$ & $1.4 \times 10^{5}$ \\
$R(\mathrm{~J} / \mathrm{mol} \mathrm{K})$ & 8.314 \\
\hline
\end{tabular}


the apparent activation energy of the deformation process during plastic flow, $R$ is the universal gas constant, $T$ is the absolute temperature and $A$ is a factor depending on the $\mathrm{Mg}$ and Si matrix solute content (see [29] and references therein). The values for these parameters are grouped in Table 1.

This rigid-visco-plastic material is coupled with the following heat transfer equations:

$$
\nabla \cdot(k \nabla T)+\dot{r}-\left(\rho c_{p} \dot{T}\right)=0,
$$

where $k$ denotes thermal conductivity, $\dot{r}$ the heat generation rate, $\rho$ the material density and $c_{p}$ the specific heat of the metal. The rate of heat generation in the aluminium billet due to plastic deformation is given by

$$
\dot{r}=\beta \boldsymbol{\sigma}: \mathbf{D},
$$

where $\beta$ represents the fraction of mechanical energy converted to heat and is assumed to be 0.9 [30]. The boundary conditions here considered are

$$
\begin{aligned}
& \boldsymbol{\sigma} \cdot \mathbf{n}=\overline{\mathbf{t}} \text { in } \Gamma_{\mathrm{t}}, \\
& \mathbf{v}=\overline{\mathbf{v}} \text { in } \Gamma_{\mathrm{v}},
\end{aligned}
$$

where $\Gamma_{\mathrm{t}}$ and $\Gamma_{\mathrm{v}}$ represent, respectively, the part of the boundary $\Gamma \equiv \partial \Omega$ where traction and velocities are prescribed. In addition, along the boundary, either temperature or heat flux are prescribed.

In order to simulate the extrusion, an initial cloud of 270 nodes was considered. The initial $\alpha$-shape of this cloud is depicted in Fig. 7. This cloud of points remains the same throughout the simulation and no addition or deletion of nodes was performed.

From the mechanical point of view, prescribed velocities were applied at the lower and rightmost parts of the boundary, taking unitary values. Slip boundary conditions were considered in the remainder of the boundary. From the thermal point of view, adiabatic boundary conditions were considered inside the die, whereas convection boundary conditions were applied at the nodes of the extrudate getting out of the die. The heat transfer coefficient between the extrudate and the air is $0.1 \mathrm{~N} \mathrm{~s}^{-1} \mathrm{~mm}^{-1}{ }^{\circ} \mathrm{C}^{-1}$, and the initial billet temperature was assumed of $773 \mathrm{~K}$.

The variational formulation of Eqs. (9) and (10) using the constitutive law given by Eqs. (11) and (12) assuming the temperature field known (computed at the previous iteration) is discretized using the NEM interpolation of the velocity field and a constant pressure in each Voronoi cell (discontinuous approximation of the pressure field) that despite the fact that it does not verify the LBB condition, no locking has been noticed until now. The resulting linear system was symmetric. In the other hand, the heat equation is solved in the updated domain where the material derivative of the temperature field is discretized along the nodal trajectories by applying the method of characteristics. The coupled model has been treated using both explicit and implicit techniques (in this last case a fixed point strategy was accounted). In all the cases stability was checked and the convergence when the number of nodes is increased, proved.

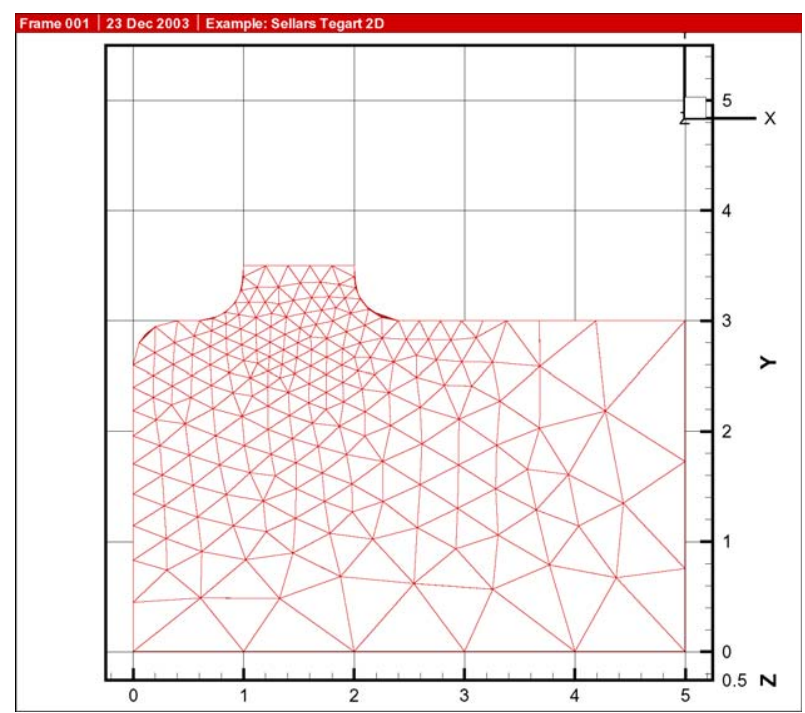

Fig. 7. Initial $\alpha$-shape of the cloud of points for the extrusion simulation. 


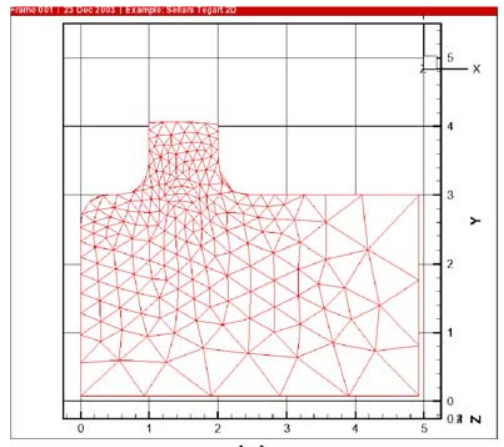

(a)

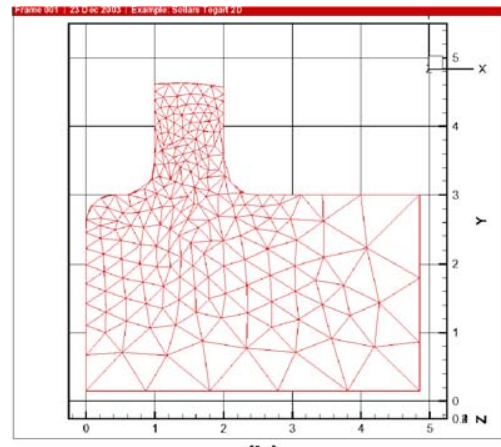

(b)

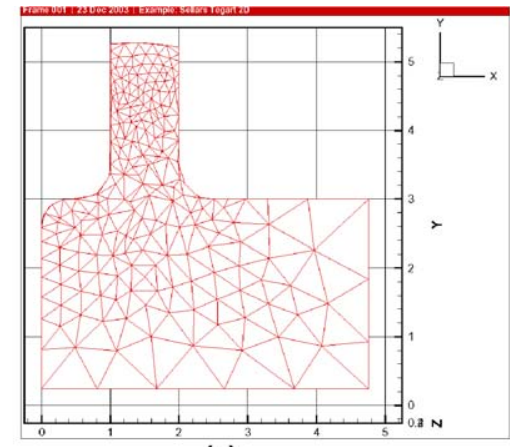

(c)

Fig. 8. Snapshots of the $\alpha$-shapes at time steps 30,60 and 96.

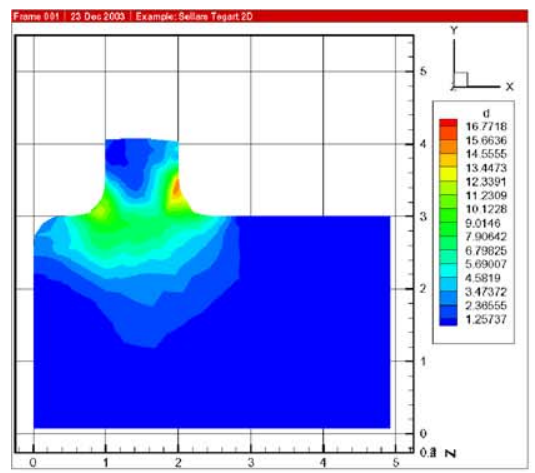

(a)

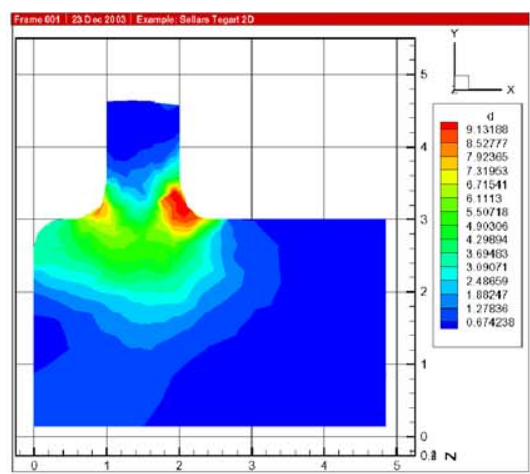

(b)

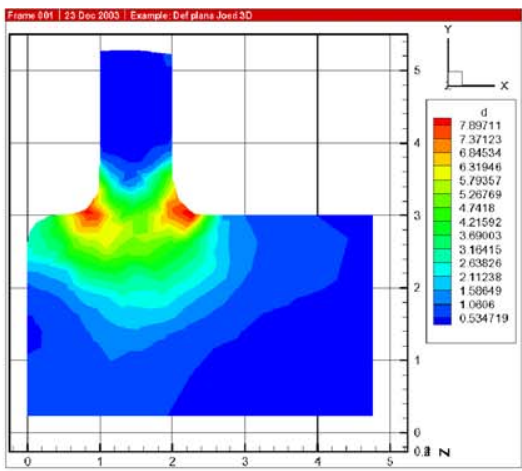

(c)

Fig. 9. Equivalent plastic strain rate for time steps 30, 60 and 96.

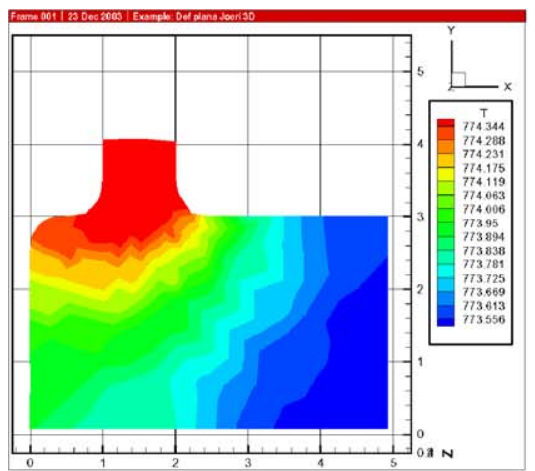

(a)

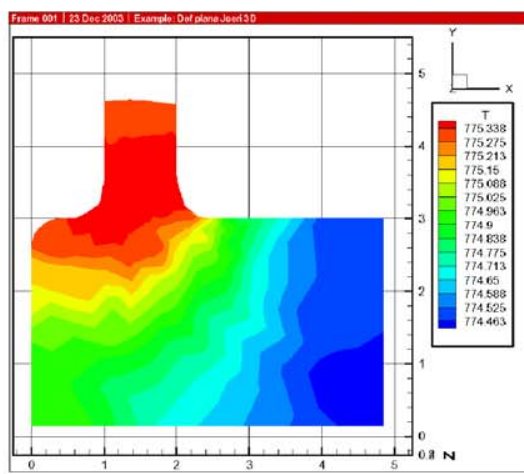

(b)

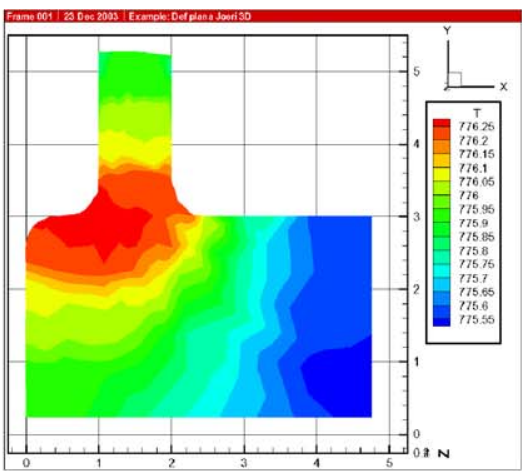

(c)

Fig. 10. Temperature field for time steps 30, 60 and 96.

The simulation runs over 96 time steps of 0.0025 s. $\alpha$-shapes for time steps 30,60 and 96 are plotted in Fig. 8 .

Equivalent plastic strain rate for time steps 30,60 and 96 is depicted in Fig. 9. Note that, as proved in [31], the "quality" of the triangulation - in a FE sense - does not affect the accuracy of the results. The temperature field at time steps 30,60 and 96 is depicted in Fig. 10.

Finally, Von Mises stresses for the same time steps are shown in Fig. 11. We can notice that as the temperature increases, due to the heat generation induced by the plastic deformation, the Von Mises stresses decrease. These results are consistent with those of Lof [32], obtained using an ALE formulation in finite elements. The velocity field, at the last time step, is depicted in Fig. 12.

These results suggest the ability of an updated Lagrangian approach for the simulation of complex extrusion processes. The use of natural neighbor approaches ensures the accuracy of the results even with poor quality triangulations. In this example, a transient extrusion process has been simulated, taking into account the temperature effects during the process. 


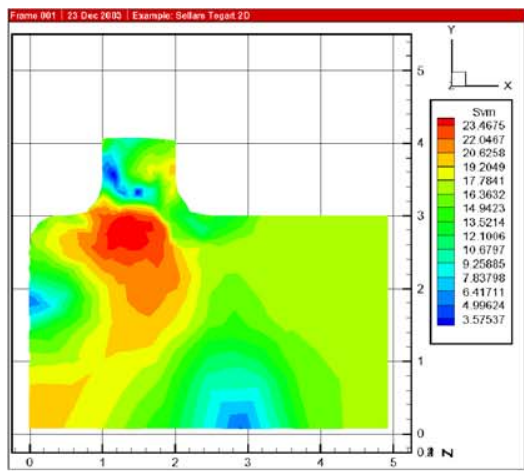

(a)

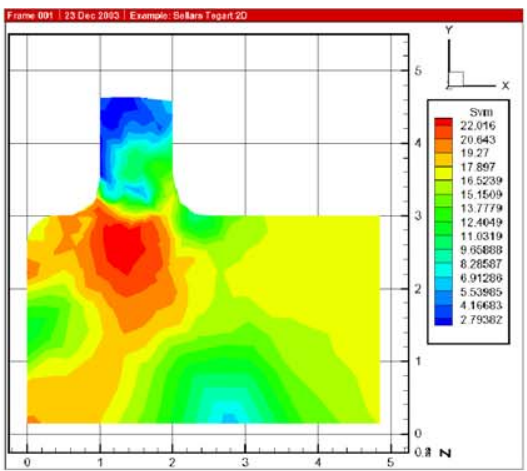

(b)

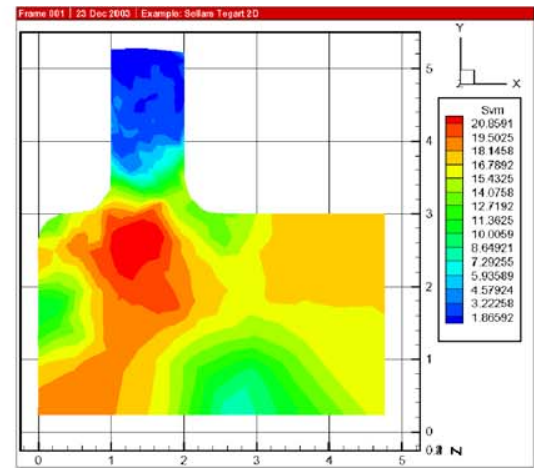

(c)

Fig. 11. Von Mises stress field for time steps 30, 60 and 96.

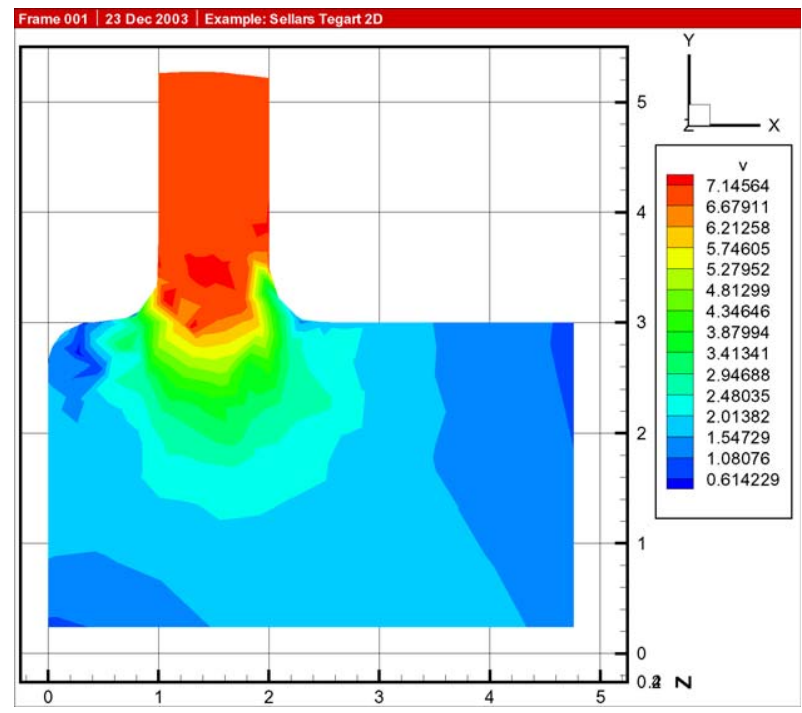

Fig. 12. Velocity field (in norm) at the final time step.

The simulation of the whole extrusion cycle, including the steady-state regime, would be an interesting goal for the technique just described.

\subsection{Metal casting simulation using the C-NEM}

Let $\Omega \in \mathfrak{R}^{2}$ be a bounded domain and $T$ the temperature field. On the domain boundary $\Gamma \equiv \partial \Omega$ the temperature or the thermal flux are prescribed. We will denote by $\Gamma_{1}$ the domain boundary where the temperature is known $T\left(\mathbf{x} \in \Gamma_{1}, t\right)=\bar{T}(\mathbf{x}, t)$ and by $\Gamma_{2}$ the domain boundary where the heat flux $\bar{q}$ is imposed. The thermal model is defined in the time interval $\left[0, t_{\max }\right]$. The initial temperature $T(\mathbf{x}, t=0)=T_{0}$, where $T_{0}$ is assumed to be higher than the material melting temperature $T_{\mathrm{m}}$. At time, $t=0$, a part of the domain boundary $\Gamma_{1}$ is suddenly submitted to a temperature $T_{1}<T_{\mathrm{m}}$. A moving solidification front $\Gamma_{\mathrm{I}}$ is then generated, whose position evolves in time, i.e. $\Gamma_{\mathrm{I}}(t)$, dividing the domain $\Omega$ in two regions $\Omega_{1}(t)$ (containing the solid phase at time $t$ ) and $\Omega_{2}(t)$ (which contains the liquid phase). For the sake of simplicity we will consider, from now on, a homogeneous and isotropic thermal model in both phases.

The heat transfer model is defined in each phase, neglecting volumetric source terms, by

$$
\begin{cases}c_{1} \frac{\partial T(\mathbf{x}, t)}{\partial t}=\nabla \cdot\left(k_{1} \nabla T\right) & \text { in } \Omega_{1}(t), \\ c_{2} \frac{\partial T(\mathbf{x}, t)}{\partial t}=\nabla \cdot\left(k_{2} \nabla T\right) & \text { in } \Omega_{2}(t),\end{cases}
$$

where $c_{1}$ and $c_{2}$ are the volumetric heat capacities of both phases, being $k_{1}$ and $k_{2}$ their thermal conductivities. The associated initial and boundary conditions are 


$$
\begin{cases}T(\mathbf{x}, t=0)=T_{0} & \forall \mathbf{x} \in \Omega, \\ T(\mathbf{x}, t)=\bar{T}(\mathbf{x}, t) & \forall \mathbf{x} \in \Gamma_{1}, \quad \forall t \in\left[0, t_{\max }\right], \\ -k \nabla T(\mathbf{x}, t) \cdot \mathbf{n}=\bar{q}(\mathbf{x}, t) & \forall \mathbf{x} \in \Gamma_{2}, \quad \forall t \in\left[0, t_{\max }\right]\end{cases}
$$

The evolution of the interface $\Gamma_{\mathrm{I}}(t)$ is described by a Stefan condition:

$$
\mathbf{V}\left(\mathbf{x} \in \Gamma_{\mathrm{I}}(t)\right)=\frac{|[q]|}{L} \mathbf{n}_{\mathbf{I}}(\mathbf{x})
$$

where $\mathbf{V}$ is the interface velocity, $L$ is the volumetric latent heat of fusion, $\mathbf{n}_{\mathbf{I}}(\mathbf{x})$ is the normal vector to the interface at point $\mathbf{x}$ which is assumed to point into the liquid phase, and $|[q]|$ the thermal flux jump across the interface $\Gamma_{\mathrm{I}}(t)$, i.e.

$$
|[q]|=\left(\left.k_{1} \nabla T\right|_{\Gamma_{\mathrm{I}}^{-}(t)}-\left.k_{2} \nabla T\right|_{\Gamma_{\mathrm{I}}^{+}(t)}\right) \mathbf{n}_{\mathrm{I}} .
$$

The additional constraint prescribed on the interface $\Gamma_{\mathrm{I}}(t)$ is

$$
T(\mathbf{x}, t)=T_{\mathrm{m}} \quad \forall \mathbf{x} \in \Gamma_{\mathrm{I}}(t),
$$

where $T_{\mathrm{m}}$ is the melting temperature.

For application to problems involving crystal growth and dentritic solidification, one would like to take into account the effects of surface tension, crystalline anisotropy and molecular kinetics. In this case the previous equation must be changed by the Gibbs-Thomson relation.

Assuming that $\Gamma_{1} \equiv \Gamma$ and that $\Gamma_{2}$ reduces to the moving interface $\Gamma_{\mathrm{I}}$, i.e. $\Gamma_{2} \equiv \Gamma_{\mathrm{I}}$, the weak formulation associated with Eq. (17) results:

Find $T \in H^{1}(\Omega)$ verifying $T=\bar{T}$ on $\Gamma_{1}$ such that:

$$
\int_{\Omega} c \frac{\partial T}{\partial t} \delta T \mathrm{~d} \Omega=-\int_{\Omega} k \nabla T \cdot \nabla \delta T \mathrm{~d} \Omega+\int_{\Gamma_{\mathrm{I}}(t)}|[q]| \delta T \mathrm{~d} \Gamma \quad \forall \delta T \in H_{0}^{1}(\Omega),
$$

where $H^{1}(\Omega)$ and $H_{0}^{1}(\Omega)$ are the usual Sobolev functional spaces. Substituting the trial and test functions (both approximated in the C-NEM framework) in the above equation and using the arbitrariness of the field $\delta T$, the following system of equations is obtained:

$$
\mathbf{C} \dot{\mathbf{T}}+\mathbf{K T}=\mathbf{F},
$$

where $\mathbf{T}$ is the vector containing the unknown nodal temperatures. We consider the solution on the time interval $\left[0, t_{\max }\right]$, partitioned into steps as $\left[t^{n}, t^{n+1}\right]$ and the generalized trapezoidal time stepping algorithm characterized by the parameter $\theta$ :

$$
\frac{\partial T^{n+1}}{\partial t}=\frac{T^{n+1}-T^{n}-(1-\theta) \Delta t \frac{\partial T^{n}}{\partial t}}{\theta \Delta t}
$$

which leads to

$$
\left(\mathbf{C}^{n+1}+\theta \Delta t \mathbf{K}^{n+1}\right) \mathbf{T}^{n+1}=\mathbf{F}^{n+1}\left(\mathbf{T}^{n},|[q]|^{n+1}\right)
$$

with

$$
\mathbf{C}^{n+1}=\int_{\Omega^{n+1}} \mathbf{N}^{t} c \mathbf{N} \mathrm{d} \Omega
$$

where $\Omega^{n+1}=\Omega_{1}^{n+1} \cup \Omega_{2}^{n+1}$;

$$
\mathbf{K}^{n+1}=\int_{\Omega^{n+1}} \mathbf{B}^{t} k \mathbf{B} \mathrm{d} \Omega
$$

and

$$
\mathbf{F}^{n+1}=\mathbf{C}^{n+1} \mathbf{T}^{n}+(1-\theta) \Delta t \int_{\Omega^{n+1}} \mathbf{N}^{t} c \frac{\partial T^{n}}{\partial t} \mathrm{~d} \Omega+\theta \Delta t \int_{\Gamma_{\mathrm{I}}^{n+1}} \mathbf{N}^{t}|[q]|^{n+1} \mathrm{~d} \Gamma,
$$

where $\mathbf{N}$ is the vector containing the nodal shape functions

$$
\mathbf{N}=\left[\begin{array}{llll}
\phi_{1} & \phi_{2} & \cdots & \phi_{N}
\end{array}\right]
$$


and $\mathbf{B}$ is the matrix containing the shape functions derivatives

$$
\mathbf{B}=\left[\begin{array}{llll}
\phi_{1, x} & \phi_{2, x} & \cdots & \phi_{N, x} \\
\phi_{1, y} & \phi_{2, y} & \cdots & \phi_{N, y}
\end{array}\right] .
$$

The stabilized conforming nodal integration proposed by Chen et al. in [33] is employed for the numerical integration of $\mathbf{K}$ (see our former work [12] for more details). A lumped mass matrix $\widetilde{\mathbf{C}}$ is computed making use of the constrained Voronoi cells areas as nodal weights.

The iteration procedure is defined as:

Knowing $\mathbf{T}^{n}$ and $|[q]|^{n}$ at time $t^{n}$, the non-linear problem associated with Eq. (25) results in finding $\mathbf{T}^{n+1}$ and $|[q]|^{n+1}$ such that Eqs. (21) and (25) are satisfied. For this purpose we proceed as follows:

1. Compute the interface velocity $\mathbf{V}^{n}(\mathbf{x})$ using Eq. (19) and update the interface position at time $t^{n+1}$ using the forward Euler formula:

$$
\mathbf{x}_{J}^{n+1}=\mathbf{x}_{J}^{n}+\Delta t \mathbf{V}^{n}\left(x_{J}^{n}\right),
$$

where $\mathbf{x}_{J}$ are the nodes defining the interface.

2. Update locally the constrained Voronoi diagram and the shape functions associated with integration points in the interface neighborhood. Then, we compute $\widetilde{\mathbf{C}}^{n+1}$ and $\mathbf{K}^{n+1}$.

3. Solve Eq. (25) using a Newton-Raphson procedure where the tangent matrix is computed numerically.

4. Repeat while $t^{n+1}<t_{\max }$.

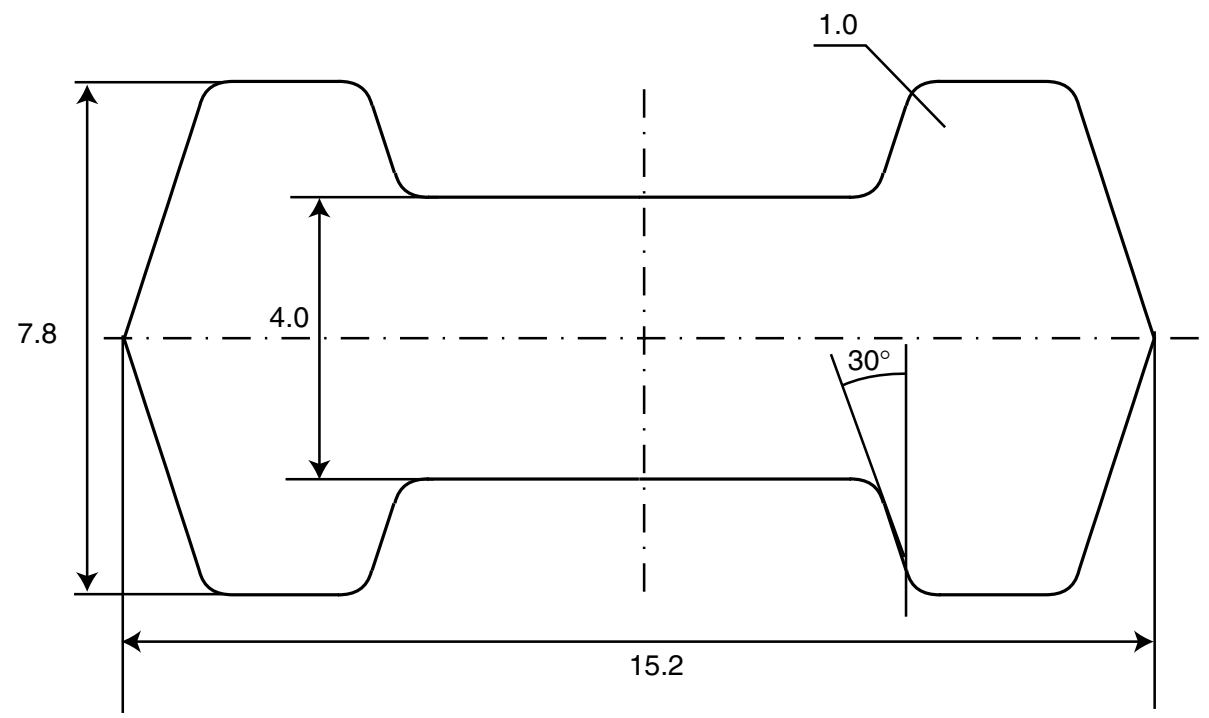

Fig. 13. Aluminium part.

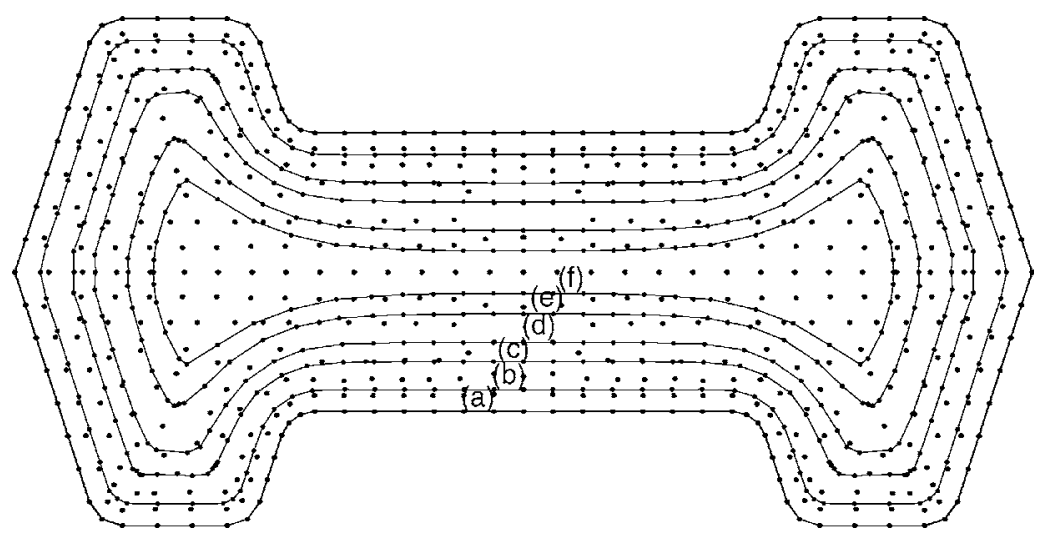

Fig. 14. Interphase location during the cooling process 


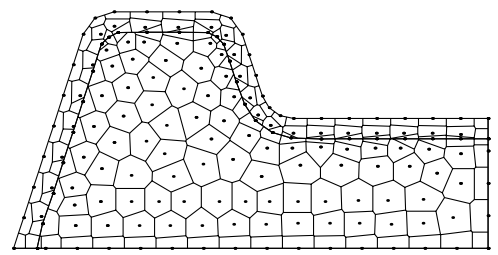

$\mathrm{t}=0.26 \mathrm{~s}$

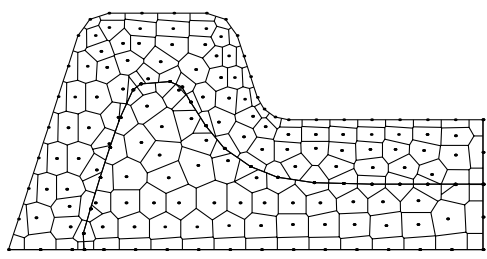

$t=2.64 \mathrm{~s}$

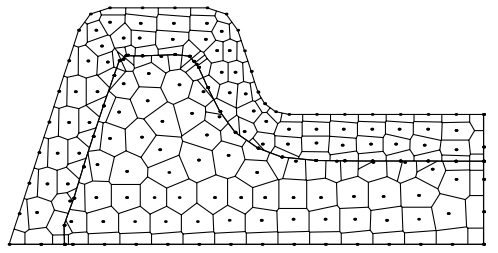

$\mathrm{t}=1.44 \mathrm{~s}$

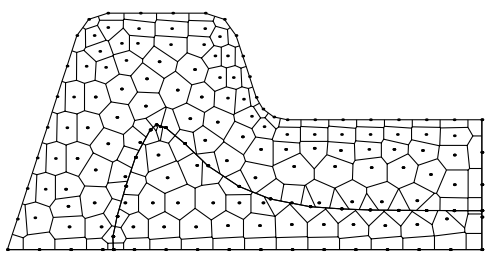

$t=7.92 \mathrm{~s}$

Fig. 15. Constrained Voronoi diagrams for four interphase positions.

Table 2

Thermal properties of aluminium [35]

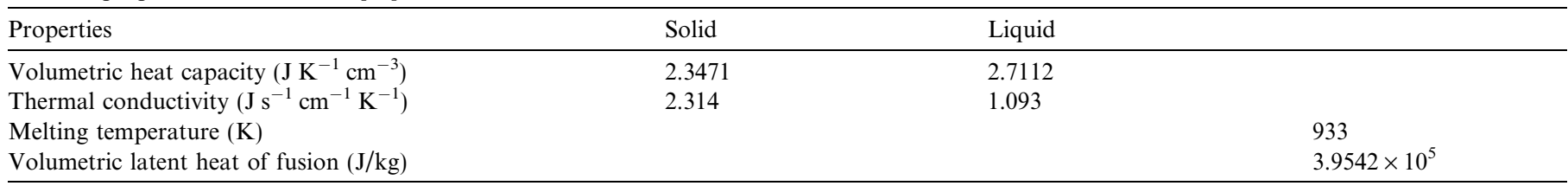

Fig. 13 (units in $\mathrm{cm}$ ) shows the considered 2D geometry, where the initial temperature is assumed equal to $1000 \mathrm{~K}$, and a temperature of $300 \mathrm{~K}$ is prescribed suddenly on the domain boundary. Fig. 14 depicts a sequence of the interphase position during the cooling process and Fig. 15 illustrates the associated constrained Voronoi diagrams. The aluminium thermal properties considered in these simulations are listed in Table 2. More simple problems, with exact solutions known, were treated in [34], where it was concluded the excellent accuracy of the C-NEM to treat the Stefan problems involving moving interphases. Moreover, this excellent accuracy does not depend significantly on the regularity of the nodes distribution. Thus, very accurate solutions were obtained despite of the highly distorted Delaunay triangulation related to the cloud of nodes. In the problem just analyzed, no reference solution exist, however, no anomalous behaviors have been noticed.

\section{Conclusions}

In this paper we have presented and discussed the application of the meshless natural element method, for simulating some forming processes involving large displacements as well as moving discontinuities. The excellent accuracy of both techniques despite of the highly distorted associated Delaunay triangles, has been proved in some test problems, whose exact solutions are known, in some of our former works [24,34]. The exact solutions related to the problems analyzed in this paper are not known, however, it must be pointed out that despite of the highly distorted triangulations associated with the cloud of nodes, no anomalous behaviors have been noticed.

The natural neighbor Galerkin method, or natural element method (NEM), satisfies the Kronecker delta property as well as the linear consistency (which implies the partition of unity). Despite these appealing properties, this approximation is linear only on the boundary of convex domains. In this paper we have presented two strategies able to recover this linearity on any boundary (convex or not). The first strategy invokes the $\alpha$-shape concept that, in addition, avoids an explicit description of the domain boundary (which is automatically extracted from the cloud of nodes), whereas the second strategy proceeds by introducing a visibility criterion, and the associated constrained Voronoi diagram.

\section{References}

[1] I. Babuška, A.K. Aziz, On the angle condition in the finite element method, SIAM J. Numer. Anal. 13 (2) (1976) $214-226$.

[2] L.B. Lucy, A numerical approach to the testing of fusion process, Astron. J. 88 (1977) 1013-1024.

[3] B. Nayroles, G. Touzot, P. Villon, Generalizing the finite element method: diffuse approximation and diffuse elements, Comput. Mech. 10 (1992) 307-318. 
[4] J.M. Melenk, I. Babuška, The partition of unity finite element method: basic theory and applications, Comput. Methods Appl. Mech. Engrg. 139 (1996) 289-314.

[5] C.A. Duarte, J.T. Oden, An h-p adaptative method using clouds, Comput. Methods Appl. Mech. Engrg. 139 (1996) $237-262$.

[6] T. Belytschko, Y.Y. Lu, L. Gu, Element-free Galerkin methods, Int. J. Numer. Methods Engrg. 37 (1994) $229-256$.

[7] W.K. Liu, S. Jun, Y.F. Zhang, Reproducing kernel particle methods, Int. J. Numer. Methods Fluids 21 (1995) $1081-1106$.

[8] J.S. Chen, C.T. Wu, Y. Yoon, New boundary conditions treatment in meshfree computation of contact problems, Comput. Methods Appl. Mech. Engrg. 187 (2000) 441-468.

[9] M. Sambridge, J. Braun, M. McQueen, Geophisical parameterization and interpolation of irregular data using natural neighbors, Geophys. J. Int. 122 (1995) 837-857.

[10] N. Sukumar, B. Moran, T. Belytschko, The natural elements method in solid mechanics, Int. J. Numer. Methods Engrg. 43 (1998) $839-887$.

[11] E. Cueto, J. Cegoñino, B. Calvo, M. Doblaré, On the imposition of essential boundary conditions in natural neighbor Galerkin methods, Commun. Numer. Methods Engrg. 19 (2003) 361-376.

[12] J. Yvonnet, D. Ryckelynck, P. Lorong, F. Chinesta, A new extension of the natural element method for non-convex and discontinuous problems: the constrained natural element method (C-NEM), Int. J. Numer. Methods Engrg. 60 (8) (2004) 1451-1474.

[13] J. Yvonnet, D. Ryckelynck, P. Lorong, F. Chinesta, Interpolation naturelle sur les domaines non convexes par l'utilisation du diagramme de Voronoi contraint: méthode des éléments C-naturels, Rev. Européene Eléments Finis 12 (4) (2003) 487-509.

[14] I. Babuška, J.M. Melenk, The partition of unity method, Int. J. Numer. Methods Engrg. 40 (1997) 727-758.

[15] M. Sussman, P. Smereka, S. Osher, A level set approach for computing solutions to incompressible two-phase flows, J. Comput. Phys. 114 (1994) 146-159.

[16] N. Sukumar, D. Chop, N. Moes, T. Belytschko, Modeling holes and inclusions by level sets in the extended finite element method, Comput. Methods Appl. Mech. Engrg. 190 (2001) 6183-6200.

[17] H. Ji, D. Chopp, J.E. Dolbow, A hybrid finite element/level set method for modeling phase transformations, Int. J. Numer. Methods Engrg. 54 (2002) $1209-1233$.

[18] J.A. Sethian, Fast marching methods, SIAM Rev. 41 (2) (1999) 199-235.

[19] D. González, E. Cueto, M. Doblaré, Volumetric locking in natural neighbour Galerkin methods, Int. J. Numer. Methods Engrg. 61 (4) (2004) 611-632.

[20] R. Sibson, A vector identity for the Dirichlet tesselations, Math. Proc. Camb. Philos. Soc. 87 (1980) 151-155.

[21] H. Hiyoshi, K. Sugura, Improving continuity of Voronoi-based interpolation over Delaunay spheres, Comput. Geomet. 22 (2002) 167-183.

[22] N. Sukumar, B. Moran, Y. Semenov, V.V. Belikov, Natural neighbor Galerkin methods, Int. J. Numer. Methods Engrg. 50 (2001) 207-219.

[23] H. Edelsbrunner, D.G. Kirkpatrick, R. Seidel, On the shape of a set of points in the plane, IEEE Trans. Inform. Theory IT-29 (4) (1983) 551-559.

[24] M.A. Martínez, E. Cueto, I. Alfaro, M. Doblaré, F. Chinesta, Updated Lagrangian free surface flow simulations with natural neighbour Galerkin methods, Int. J. Numer. Methods Engrg. 60 (13) (2004) 2105-2129.

[25] E. Cueto, B. Calvo, M. Doblare, Modeling three-dimensional piece-wise homogeneous domains using the $\alpha$-shape based natural element method, Int. J. Numer. Methods Engrg. 54 (2002) 871-897.

[26] J.R. Shewchuck, Tetrahedral mesh generation by delaunay refinement, in: Proceedings of the Fourteenth Annual Symposium on Computational Geometry, Minneapolis, MN, June 1998, pp. 86-95.

[27] J.R. Shewchuck, Sweep algorithms for constructing higher-dimensional constrained Delaunay triangulations, in: Proceedings of the Sixteenth Annual Symposium on Computational Geometry, Hong-Kong, June 2000, pp. 350-359.

[28] C.M. Sellars, W.J.M. Tegart, La relation entre la résistance et la structure dans la déformation à chaud, Mem. Sci. Rev. Met. 63 (1966) $731-746$.

[29] J. Lof, Y. Blokhuis, FEM simulations of the extrusion of complex thin-walled aluminium sections, J. Mater. Process. Technol. 122 (2002) $344-354$.

[30] J. Zhou, L. Li, J. Duszczyk, 3D FEM simulation of the whole cycle of aluminium extrusion throughout the transient state and the steady state using the updated Lagrangian approach, J. Mater. Process. Technol. 134 (2003) 383-397.

[31] N. Sukumar, B. Moran, T. Belytschko, The natural element method in solid mechanics, Int. J. Numer. Methods Engrg. 43 (5) (1998) $839-887$.

[32] J. Lof, Elasto-viscoplastic FEM simulation of the aluminium flow in the bearing area for extrusion of thin-walled sections, J. Mater. Process. Technol. 114 (2001) 174-183.

[33] J.S. Chen, C.T. Wu, Y. Yoon, A stabilized conforming nodal integration for Galerkin mesh-free methods, Int. J. Numer. Methods Engrg. 50 (2001) 435-466.

[34] J. Yvonnet, F. Chinesta, Ph. Lorong, D. Ryckelynck, The constrained natural element method (C-NEM) for treating thermal models involving moving interfaces, Int. J. Therm. Sci. 44 (6) (2005) 559-569.

[35] E.A. Brandes, Smithells Metals Reference Book, Butterwoths and Co., Ltd., London, 1983. 